\title{
Sacral Fracture after Posterior Screw Fixation: A Case Report
}

\author{
Yun-Young Park, Young Gyu Kim, Mou Seop Lee, Kyung Soo Min, Young Seok Park, Jong Beom Lee \\ Department of Neurosurgery, Chungbuk National University Hospital, Chungbuk National University College of Medicine, Cheongju, \\ Republic of Korea
}

\begin{abstract}
Sacral fractures and failures are possible complication of instrumented lumbosacral arthrodesis. And it has received increasing attention in the surgical literature. It is sometimes difficult to diagnose the fractures and make timely treatment. It is helpful that preoperative awareness of risk factors involved sacral fracture and spinopelvic parameters in making plan of treatment and prevention of postoperative complication. There are many possible complications after posterior screw fixation, such as neurological or vascular injury, loss of curve correction, intraoperative pedicle fracture or loosening, dura injury, deep infection, and pseudarthrosis. But sacral fracture, distal part of lumbosacral arthrodesis is not common case. In this paper, we describe a case of sacral fracture of distal level of fixed vertebral body after lumbosacral arthrodesis in old aged woman and assessment of risk factors. The patient had herniated nucleus pulposus at L5/S1 level, and discectomy is operated. After operation, the symptom has relieved, but 1-year later other symptoms recurred radiating pain on both leg and back pain due to bilateral foraminal stenosis and central stenosis. So, lumbosacral arthrodesis and posterior screw fixation to L4-5-S1 levels are operated. Finally, the symptoms are resolved, but after spine fusion operation 1-month, it was found that sacral fracture of S1 vertebral body, distal part of spine fixation. The conservative treatment is chosen, and there is no aggravation of dislocation or deformity in follow-up exam. If the deformity aggravates, we make plan to surgery, extension of screw fixation to iliac bone.
\end{abstract}

Key Words: Adult spine deformity; Malnutrition; Posterior spine arthrodesis; Sacral fracture

$\triangle$ Corresponding Author: Jong Beom Lee, Department of Neurosurgery, Chungbuk National University Hospital, Chungbuk National University College of Medicine, Chungdae-ro 1, Seowon-Gu, Cheongju 28644, Republic of Korea Tel: +82-43-269-6087, Fax: +82-43-273-1614, E-mail: miraenobelist@gmail.com

\section{INTRODUCTION}

As the society is older aged, the number of patients of degenerative spine disease such as multilevel spinal stenosis is increased. Because the spine instrumentation surgery with wide decompression is effective treatment for degenerative spine disease, the number of spine surgery is increased, continuously ${ }^{1)}$.

Nevertheless, there are some complications after posterior screw fixation such as neurological or vascular injury, loss of curve correction, intraoperative pedicle fracture or loosening, dura injury, deep infection, and pseudarthrosis ${ }^{2}$.

Because the old aged patients have high prevalence of osteoporosis, malnutrition, and often use long-term steroid, they are high-risk group of any fracture. But, the postoperative sacral fracture, distal part of lumbosacral arthrodesis, is rare and its pain is sometimes masked with postoperative pain, and that makes difficult early detection of sacral complication.

In a case of sacral fracture of distal level of fixed vertebral body after lumbosacral arthrodesis in old aged, we suggest the diagnosis and treatment of the fracture.

\section{CASE REPORT}

An 83-year-old female patient, previously operated L5/S1 level discectomy for leg pain. After 1- year previous operation, the patient complained lower back pain and recurred radiating pain on both legs, and did not be relieved although medical pain management and physical therapy programs. After lying down for 2-month at local clinic, she revisited our hospital for treatment. The plain radiographs and magnetic resonance images show spinal stenosis for multiple level, and spondylolisthesis of L3-4 level (Fig. 1). There is an evidence of osteopenia, on Bone DEXA scan. We operated her transforaminal lumbar interbody fusion for L4-5-S1 level, L3 laminectomy, posterior screw fixation for L3-4-5-S1 levels. There is no complication, intraoperative and post-operative.

After spine surgery, she felt the pain relieved, so started ambulation and rehabilitation therapy. But 1-month after 

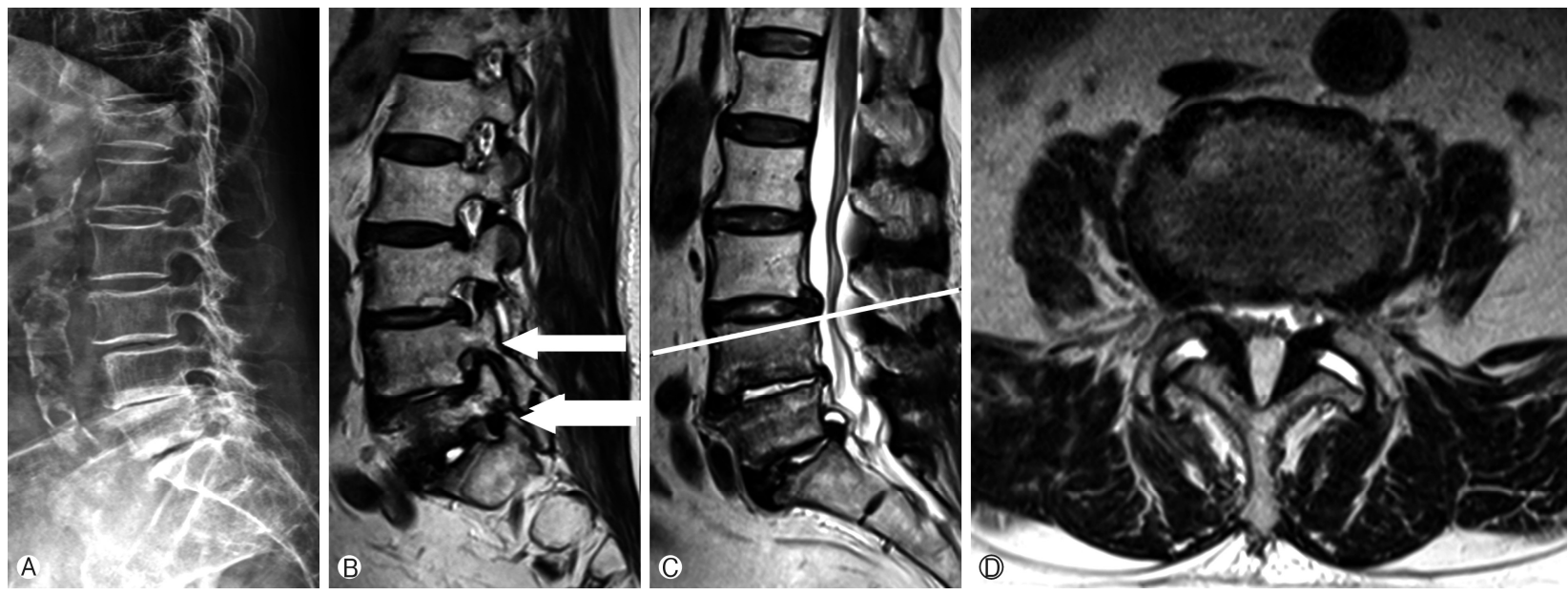

Fig. 1. Preoperative simple radiograph and MR images. (A) There is G1 anterolisthesis on L3-4 level and disc space narrow and multiple vacuum discs. (B) And there are foraminal spinal stenosis on L4-5-S1 levels (white arrow). (C, D) There are central spinal stenosis due to liga- mentum flavum hypertrophy and bulging disc on L3-4. And there is severe facet arthropathy, bilateral.

ambulation, moderate low back and buttock pain occurred. The X-ray and CT images showed ' $U$ ' sacral fracture, classified Roy-Camille type 2 anterior displacement (Fig. 2). We suggested bed rest for 1-month for treatment of sacral fracture, and medical treatment of osteopenia and malnutrition state, so the pain is reduced and there is no evidence of further displacement of fracture.

So, the patient discharged, we check the serial X-ray examination for L-S spine in follow-up visit clinic, there is no evidence of aggravation (Fig. 3).

\section{DISCUSSION}

The lumbosacral arthrodesis is an effective way to manage spine degenerative disorders and deformities, vertebral fractures, and metastatic diseases ${ }^{3-7}$. However, in the patient having low bone density, such as osteopenia, osteoporosis, it is occurred failure of fusion, pullout of pedicle screw, even the fracture of pedicle screws or vertebral body of proximal or distal part of fixed vertebrae are uncommon ${ }^{8-10)}$. In this case, the patient's BMD was osteopenia but her bone quality was very poor. For 2-month before surgery and a week after spine surgery, the patient got a bed rest. So, sarcopenia state thought to be a one of the risk factors for sacral fracture. Besides, ambulation and rehabilitation are conducted carefully, sacral fracture was occurred.

Considering the reason that the patient had sacral fracture, we thought some hypothesis. Because of poor oral intake after surgery, the insufficient nutrition has influence of low bone density and probability of bone fusion. Malnutrition patient has more postoperative complications include reoperation ${ }^{9)}$. Preoperative serum albumin levels have been an established marker for overall nutrition, and hypoalbuminemia measured by an albumin level less than $3.5 \mathrm{~g} / \mathrm{dL}$ can be used as a proxy for malnourishment ${ }^{11)}$. The patient's initial albumin level was $2.8 \mathrm{~g} / \mathrm{dL}$ and weight loss was $6 \mathrm{~kg}$ during 2 -month $(10 \%$ of total body weight). We recommend nutrition supply, strengthening muscles and maintaining weight through exercise before surgery. This theory is supported by published studies showing an independent association between recent weight loss resulting in malnutrition and an increased risk for postoperative complications ${ }^{12)}$.

Obesity, multi-segmental lumbosacral fusion, mismatched lumbar lordosis (LL)- pelvic incidence (PI) and high PI are also risk-factors of postoperative sacral fracture ${ }^{13)}$. There was no PI to LL mismatch, postoperatively.

There are various treatments have been reported in the literature. These include conservative management, initial conservative treatment followed by surgical treatment after failure of conservative treatment, and primary surgical treatment. But there is no consensus on optimal treatment for sacral fracture after lumbosacral fusion. Three out of four cases reported by Vavken et al. were treated conservatively with good result ${ }^{14)}$. In a radiographic study conducted by Wilde et al., 11 of 23 fractures were treated nonoperatively, and the other 12 went on to surgery with transiliac fixation ${ }^{15}$. Because there is similar improvement rate between surgical and conservative treatment ${ }^{16}$. The patient was tolerable for immobilization and pain. There was no neurological deficit and significant displacement. So, considering the re-operation risk of the patient we make a treatment plan conservative treatment by medical treatment of osteopenia and malnutrition state. And bed side physical treatment worked together by rehabilitation team.

As masked with postoperative pain, it is difficult to dia- 

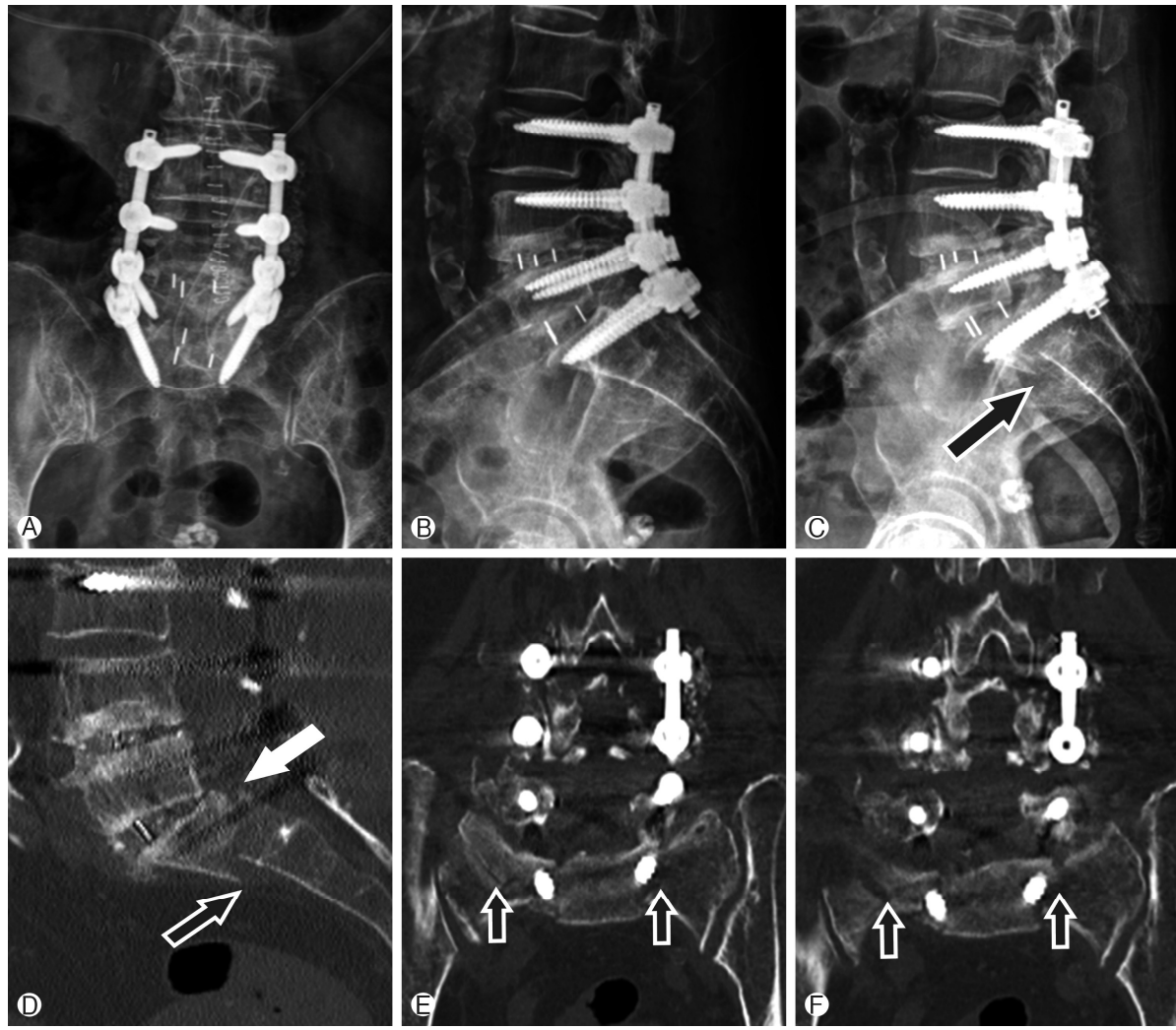

Fig. 2. (A, B) Post-operative simple radiograph findings, anterior- posterior and lateral views. There are TLIF, L4-5-S1 with posterior screw fixation, L3-4-5-S1. (C, D) One months after ambu- lation, moderate low back and buttock pain occurred. The simple radiograph and CT images showed sacral fracture (black arrow). The posterior margin is not misalignment (white arrow). ( $E, F)$ The coronal view of post-operative CT images of sacrum. There is a 'U' fracture (black arrow), classified as Roy-Camille type 2 - anterior displacement.
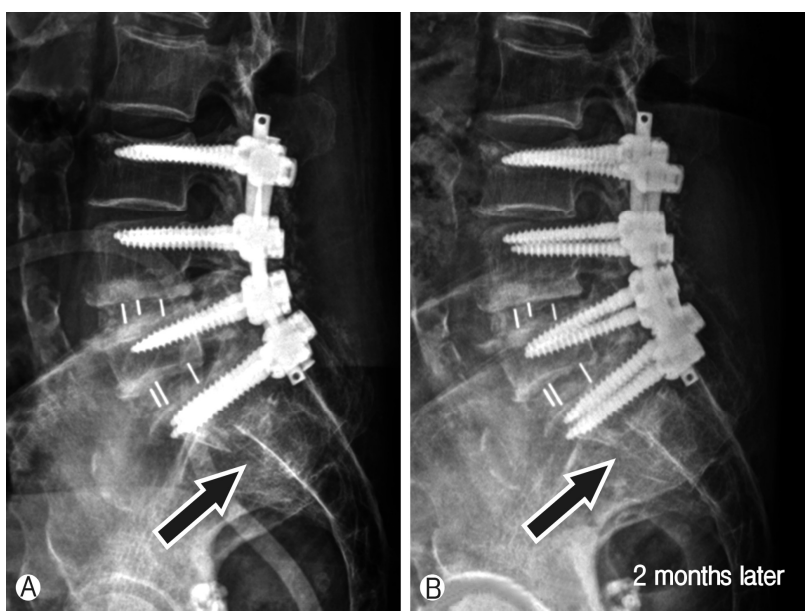

Fig. 3. (A, B) The lateral simple radiograph follow-up on 2-month after sacral fracture occured. There are no aggravation of fracture and dislocation (black arrow). And no evidence of neurologic symptoms. So, conservative management is chosen instead of surgical treatment. gnose postoperative sacral fracture, especially around the operation site. Therefore, it should be performed CT or MR images when patients complain of renewed lower back pain or buttock pain.

After occurring sacral fracture, we checked the serial simple radiograph, CT scan and estimated patient symptom. In the present patient did not opted for additional surgery because pain and disability were mild. Most importantly, if the risk factors are corrected before operation, we predict the probability of fracture decreased.

\section{CONCLUSION}

After spine surgery, sacral fracture of distal fusion part is not common. To diagnosis sacral fracture, the physician should pay attention to complain of pain. If the sacral fracture is dia gnosed, the physician can choose surgical treatment or medical conservative management. Such as this patient, old-aged, having lots of underlying disease, we suggest medical conservative 
management instead of surgery. And it may be accompanied the sufficient nutrition, treatment of osteopenia and physical therapy.

\section{REFERENCES}

1. R. Kalff, C. Ewald, A. Waschke, L. Gobisch, C. Hopf: Degenerative lumbar spinal stenosis in older people current treatment options. Dtsch Arztebl Int 110(37):pp613-624, 2013

2. C. Bellabarba, T.A Schildhauer, A.R. Vaccaro, J.R Chapman: Complications associated with surgical stabilization of high-grade sacral fracture dislocations with spino-pelvic instability. SPINE 31:pp580-588, 2006

3. Bess RS, Lenke LG, Bridwell KH, et al.: Comparison of thoracic pedicle screw to hook instrumentation for the treatment of adult spinal deformity. Spine 32:555-561, 2007

4. Kim YJ, Lenke LG, Cho SK, et al.: Comparative analysis of pedicle screw versus hook instrumentation in posterior spinal fusion of adolescent idiopathic scoliosis. Spine 29:2040-2048. 2004

5. Hirabayashi S, Kumano K, Kuroki T. Cotrel-Dubousset pedicle screw system for various spinal disorders. Merits and problems. Spine.;16:1298-1304. 1991

6. Yuan HA, Garfin SR, Dickman CA, et al.: A historical cohort study of pedicle screw fixation in thoracic, lumbar, and sacral spinal fusions. Spine 19(suppl):2279S-2296S, 1994

7. Gaines RW Jr.: The use of pedicle-screw internal fixation for the operative treatment of spinal disorders. J Bone Joint Surg 82-A: 1458-1476, 2000

8. Coscia, Michael F: Pediatric chance fracture associated with pedicle screw use: A case report. Spine 22(22):2698-2701, 1997

9. $\mathrm{Hu}$, Xiaobang and Isador H. Lieberman: Proximal instrumented vertebral body chance fracture after pedicle screw instrumentation in a thoracic kyphosis patient with osteoporosis. Clinical Spine Surgery 28(1):31-36, 2015

10. Levine, David S., Jeffrey R. Dugas, Scott J. Tarantino and Oheneba Boachie-Adjei: Chance fracture after pedicle screw fixation: a case report. Spine 23(3):382-385, 1998

11. A. J. Schoenfeld, P. A. Carey, A. W Cleveland $3^{\text {rd }}$ J. O Bader, C. $\mathrm{M}$ Bono: Patient factors, comorbidities, and surgical characteristics that increase mortality and complication risk after spinal arthrodesis: A prognostic study based on 5,887 patients. Spine J 13(10):1171-1179, 2013

12. Bozzetti F, Gianotti L, Braga M, Di Carlo V, Mariani L.: Postoperative complications in gastrointestinal cancer patients: The joint role of the nutritional status and the nutritional support. Clin Nutr 26:698-709, 2007.

13. Odate, Seiichi, MD; Shikata, Jitsuhiko, MD, PhD; Kimura, et, al.: Sacral fracture after instrumented lumbosacral fusion. Spine 38:pp223-229, 2013

14. P. Vavken, P. Krepler: Sacral fractures after multi-segmental lumbosacral fusion: A series of four cases and systematic review of literature. Eur Spine J Suppl 2:S285-290, 2008

15. G. E. Wilde, T. T. Miller, R. Schneider, F. P Girardi: Sacral fractures after lumbosacral fusion: A characteristic fracture pattern. AJR Am J Roentgenol 197(1):184-188, 2011

16. Carlo Bellabarba, Thomas A Schildhauer, Alexander R Vaccaro, Jens R Chapman: Complications associated with surgical stabilization of high-grade sacral fracture dislocations with spinopelvic instability. Spine 31:pp580-588, 2006 